\title{
Trends in Demand for Acute Medical Care at Two Football Clubs over an Eighteen-Year Period
}

\author{
Thomas P. Heinink, ${ }^{1}$ Andrew W. Fogarty, ${ }^{2}$ and Matthew D. Wiles ${ }^{3}$ \\ ${ }^{1}$ Department of Anaesthesia \& Intensive Care, Derby Hospitals NHS Foundation Trust, Royal Derby Hospital, \\ Uttoxeter Road, Derby DE22 3NE, UK \\ ${ }^{2}$ Department of Respiratory Medicine, Nottingham University Hospitals NHS Trust, City Hospital Campus, \\ Hucknall Road, Nottingham NG5 1PB, UK \\ ${ }^{3}$ Department of Anaesthesia, Sheffield Teaching Hospitals NHS Foundation Trust, Royal Hallamshire Hospital, \\ Glossop Road, Sheffield S10 2JF, UK
}

Correspondence should be addressed to Thomas P. Heinink; tomheinink@doctors.org.uk

Received 28 April 2014; Accepted 15 August 2014; Published 3 September 2014

Academic Editor: Patrick Schober

Copyright (C) 2014 Thomas P. Heinink et al. This is an open access article distributed under the Creative Commons Attribution License, which permits unrestricted use, distribution, and reproduction in any medium, provided the original work is properly cited.

\begin{abstract}
Introduction. Following the Hillsborough disaster, there is a requirement for crowd doctors to be present during football matches. However, there are little data on long-term trends in utilisation of crowd doctor services at sporting events. Methods. A retrospective service evaluation of presentations to the crowd doctor for 18 consecutive English Football League seasons: 13 seasons at Northampton Town Football Club (NTFC) followed by five seasons at Leicester City Football Club (LCFC). Data were analysed to determine the reason for, and severity of, presentation and the magnitude of the intervention required. Results. There were 429 recorded presentations to the crowd doctor over the 18 seasons. Patients' age ranged from 3 to 93 years. $76 \%$ of presentations were due to an injury or illness arising at the match. The majority of presentations were with minor ailments (76\%) requiring only minor intervention (88\%); there were a small number of life-threatening presentations (1\%) including three cardiac arrests. Conclusion. Crowd doctors may see patients in all age groups, presenting in a variety of ways. Most patients will have minor symptoms, but occasionally patients present with life-threatening problems. The crowd doctor must be competent assessing and treating all of these potential patient groups and pathologies.
\end{abstract}

\section{Introduction}

Following the Hillsborough disaster in 1989 where 96 football supporters lost their lives, the Gibson report [1] was published which recommended the institution of standards for medical cover at football stadia. The guidelines stated that a suitably trained crowd doctor should be present at every match where the crowd is expected to exceed 2000 spectators, with the additional need for an ambulance in attendance for matches with a crowd of 5000 or more. Despite these recommendations, nine years after the publication of the Gibson report almost half of crowd doctors working at Scottish football stadia had not attended relevant resuscitation courses [2].

There is now increased focus on the need for adequate medical cover at mass event gatherings, with the standards of the medical cover for professional football matches now being applied to all competitive sporting fixtures attended by spectators [3]. This guidance emphasises the importance of risk assessment in the provision of spectator safety, but there is little data regarding the utilisation of crowd doctor services at sporting events and, in particular, whether the standards set over 20 years ago are still adequate for demands today.

During the season 2011/2012, almost 35 million spectators attended matches in the four divisions of English League football. The Sports Grounds Safety Authority (SGSA) is the statutory body responsible for overseeing spectator safety at football stadia within the United Kingdom (UK) and they publish annual data regarding rates of presentations to doctors at football stadia across the UK, classified by 
division [4]. For the season 2011/2012 the figures were 1236 presentations at a rate of 0.36 presentations per 10,000 spectators.

The most comprehensive data regarding utilisation of medical services at football grounds is an analysis of six consecutive seasons at Millwall Football Club, where there were 3.1 presentations per 10,000 spectators [5]. This figure includes reviews by the crowd doctor, crowd nurse, and St. John Ambulance. Similar data collection from single seasons at Aston Villa Football Club [6] and Glasgow Celtic Football Club [7] found lower attendance rates of 1.0 and 0.95 per 10,000 spectators, respectively, despite having significantly greater crowd numbers.

There is a paucity of data looking solely at the utilisation of crowd doctors and in particular how this may have changed over time. This limited amount of data makes planning medical care for football clubs at a strategic level difficult, as it is unclear precisely how many crowd doctors are required for a given crowd size and what interventions they may be required to perform.

The aims of this study were to describe the frequency of and the underlying reason for presentation to a crowd doctor at a football match. In addition, analysis was performed to assess whether there had been any changes in attendance patterns over the 18 -season period studied.

\section{Methods}

Assent was obtained from both clubs for data analysis to take place. This study was deemed to be a service evaluation, as defined by the Health Research Agency, and as such ethical approval was not sought [8].

2.1. Setting. The authors, in conjunction with other doctors, worked as crowd doctors at both Northampton Town FC (NTFC) (seasons 1995-1996 to 2007-2008) and Leicester City FC (LCFC) (seasons 2008-2009 to 2012-2013). The NTFC stadium has a maximum capacity of 7,653 spectators, whilst the LCFC stadium is larger, with a capacity of 32,262 spectators. For both stadia on any match day one crowd doctor was in attendance, working in conjunction with the St. John Ambulance and two to four paramedics from the East Midlands Ambulance Service (EMAS), all based within the club's fully equipped first-aid room. Following every consultation with a crowd doctor an entry was made into a notebook, stored in a secure on-site location. This entry included basic demographic data (name, sex, and date of birth) along with details of the presenting complaint, examination findings, treatment administered, and discharge destination.

2.2. Data Collection. Data for the time period at which the authors worked as crowd doctors at each club were analysed. This comprised 13 consecutive seasons at NTFC (1995/96 to 2007/08) and five consecutive seasons at LCFC (2008/ 2009 to 2012/2013). Data for other events, such as preseason friendly matches and nonfootball related events such as music concerts and rugby matches, were excluded from the analyses due to this potentially representing a different cohort of spectators. The analysed data only included patients assessed by the designated crowd doctor, rather than presentations dealt with solely by other healthcare providers such as the St. John Ambulance and EMAS.

The medical records were analysed to determine if the presentation was due to a new health issue related to attendance at the match, an exacerbation of a preexisting chronic condition or an opportunistic presentation that would have required the patient to consult another doctor had they not been at the match. The specific cause of the presentation was then determined and classified appropriately. The severity of the presentation was then determined and classified according to the system described by Bhangu et al. [6] as either minor (defined as requiring basic assessment and treatment only), intermediate (more advanced diagnosis or treatment required), or major (potentially life or limb threatening condition). The magnitude of the intervention required was also classified using the minor (minimal intervention, for example, provision of analgesia or dressings to wounds), intermediate (e.g., intravenous drug administration or electrocardiogram recording), and major (life-saving treatment) system. The discharge destination of the patient was also recorded. Data classification was performed by one author (Thomas P. Heinink). In order to ensure consistency, a random sample of 200 records was coclassified by a second author (Matthew D. Wiles) to ensure agreement in cause of presentation, severity of presentation, and the magnitude of the intervention. The data were entered into a Microsoft Excel spreadsheet (Version 12, Microsoft Corporation, Redmond, Washington, USA).

Match attendance figures were obtained from the respective clubs websites $[9,10]$. Data for NTFC were only available back to season 2001/2002. The mean number of medical presentations per game and the rate of medical presentations per 10,000 spectators were calculated. All other data are presented as number (percentage) or median (range).

\section{Results}

Figures 1, 2 show the trend in the mean number of presentations to the crowd doctor per game for NTFC and LCFC. It can be seen that at NTFC this number remained largely constant at mean of 0.6 presentations per game (range 0.38 1.22), except for season 1998/1999 when the number doubled. At LCFC the mean number of presentations per match was 1.54 (range 1.36-1.79). When these figures are recalculated per 10,000 spectators to take into account variation in attendance figures at different matches (Figures 3,4), the mean number of presentations per 10,000 spectators at NTFC was 0.98 (range $0.68-1.46$ ) and 0.72 (range $0.59-0.98$ ) per 10,000 at LCFC. These rates have been relatively consistent at both clubs. These data only include 12 seasons as attendance figures were unavailable for NTFC prior to the 2000/2001 season.

Overall 429 presentations to the crowd doctor were recorded, 226 (53\%) at NTFC and 203 (47\%) at LCFC. Of these patients, 296 (69\%) were male, 125 (29\%) were female, and no gender was recorded for eight patients (2\%). These proportions were similar when the clubs were considered separately. Of the 226 presentations at NTFC, 82 (36\%) were 


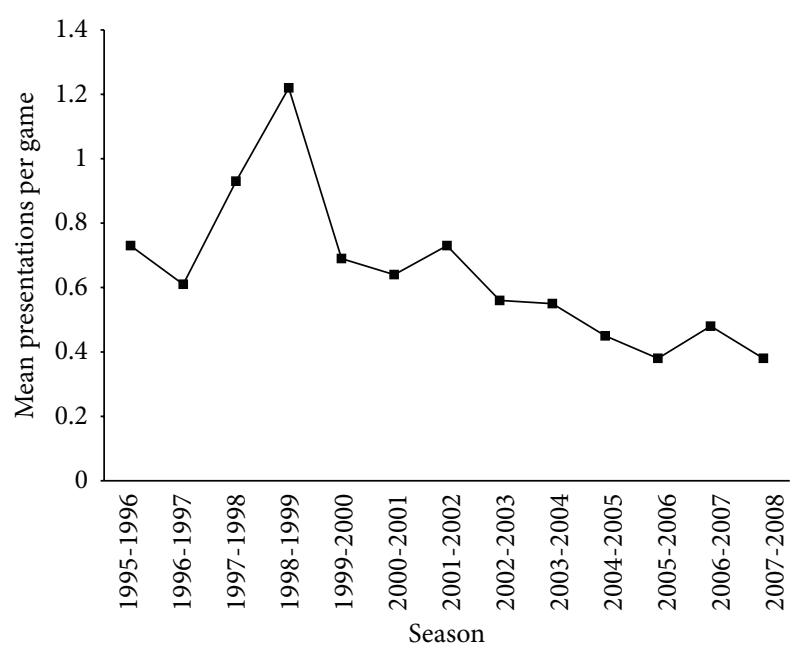

FIGURE 1: Mean number of presentations to the crowd doctor per game over the study period for NTFC.

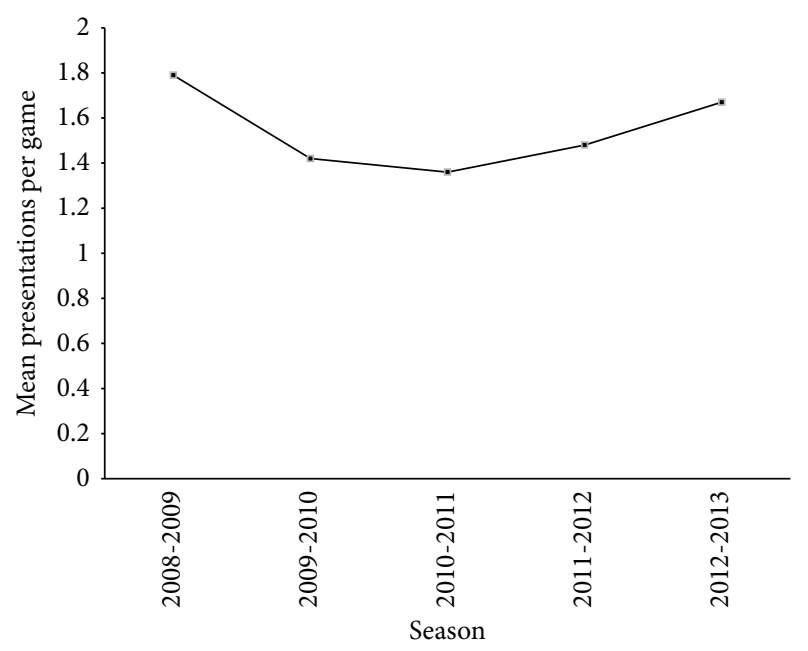

Figure 2: Mean number of presentations to the crowd doctor per game over the study period for LCFC.

adults and $44(20 \%)$ were children ( $<18$ years of age), whilst 100 (44\%) had no age recorded. At LCFC 137 (68\%) were adults and $56(28 \%)$ children, and $10(5 \%)$ patients had no age recorded. The median age for both clubs was 28 years, with a range of 4-93 years at NTFC and 3-92 years at LCFC.

Alcohol was recorded as having been a contributing factor in $8(4 \%)$ cases at NTFC and $18(9 \%)$ cases at LCFC.

Of the 226 presentations to the crowd doctor at NTFC, $170(75 \%)$ were deemed to be due to a new condition arising whilst at the stadium, 43 (19\%) were due to exacerbations of preexisting medical conditions, and $12(5 \%)$ were felt to have been opportunistic presentations of conditions not related to the patient's attendance at the football stadium. One patient presented with an obstetric condition. Of the new presentations, $93(55 \%)$ were as a result of traumatic injuries compared to $77(45 \%)$ with medical conditions.

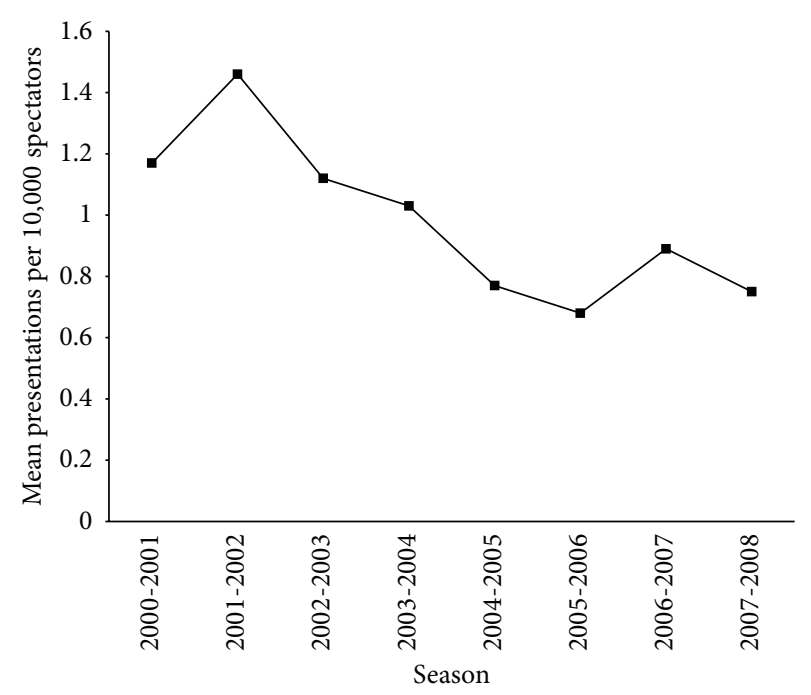

Figure 3: Mean number of presentations to crowd doctors per 10,000 spectators over the study period for NTFC.

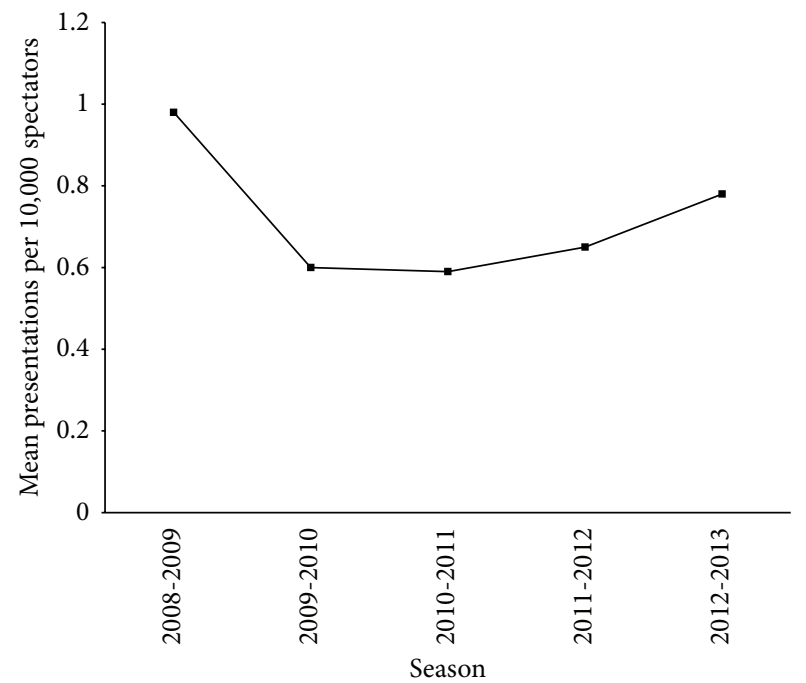

Figure 4: Mean number of presentations to crowd doctors per 10,000 spectators over the study period for LCFC.

At LCFC, the corresponding figures were 155 (76\%) new presentations, 31 (15\%) exacerbations of preexisting conditions, and $17(8 \%)$ opportunistic presentations. Of the new presentations, 97 (63\%) were due to trauma and $58(37 \%)$ to medical problems.

Table 1 summarises the causes of presentations to the crowd doctor at both NTFC and LCFC. At both clubs, the most common reason for presentation was as a result of minor soft tissue injuries and head injuries. At NTFC the joint most common reason for presenting was with a headache. Patients with new cardiac problems, predominantly chest pain or arrhythmias, made up a significant minority of the patients at both clubs. The crowd doctor also attended three cardiac arrests, one at NTFC and two at LCFC. Two of these patients were successfully resuscitated and transferred to 
TABLE 1: Presenting complaint resulting in review by crowd doctor.

\begin{tabular}{lccc}
\hline \multirow{2}{*}{ Cause of presentation } & \multicolumn{3}{c}{ Number of presentations } \\
& Northampton & Leicester & Total \\
\hline Trauma & 27 & 27 & 54 \\
Soft tissue injuries & 20 & 30 & 50 \\
Head injuries & 12 & 9 & 21 \\
Fractures or dislocations & 10 & 13 & 23 \\
Lacerations & 24 & 18 & 42 \\
Other & & & \\
Medical & 27 & 4 & 31 \\
Headaches & 8 & 14 & 22 \\
Cardiac problems & 10 & 8 & 18 \\
Infective symptoms & 4 & 9 & 13 \\
Seizures and faints & 1 & 2 & 3 \\
Cardiac arrest & 27 & 21 & 48 \\
Other & & & \\
Exacerbations & 16 & 7 & 23 \\
Musculoskeletal & 5 & 9 & 14 \\
Respiratory & 3 & 1 & 4 \\
Cardiac & 19 & 14 & 33 \\
Other & & & \\
Opportunistic & 8 & 6 & 11 \\
Preexisting trauma & 5 & & \\
Other & & & \\
\hline
\end{tabular}

hospital, whilst one was pronounced dead at the stadium. Figures 5, 6 show how the cause of presentation has changed over each season at each club.

Table 2 shows the severity of both the reason for presentation and the resultant intervention. It can be seen that the majority of patients presented with minor complaints which required minimal treatment. The major interventions performed included life-saving measures such as cardiopulmonary resuscitation (CPR), tracheal intubation, and the initial treatment of presumed myocardial infarction.

Table 3 shows the discharge destination of the patients. The majority of patients returned to the match or returned home and required no further assessment or treatment. 5\% of patients at each club were able to return to the match but were advised to attend the emergency department later the same day for treatment of urgent, but non-life-threatening, conditions (e.g., for radiological imaging of possible hand fractures).

\section{Discussion}

This is the longest continuous published dataset of demand for acute medical care in the English Football league. Data analysis of 18 seasons of data from NTFC and LCFC reveals that the majority of presentations to the crowd doctors were with minor traumatic injuries, predominantly soft tissue and head injuries. A significant minority of presentations were related to spectators' preexisting medical conditions. It also appeared that a minority of spectators use the ready

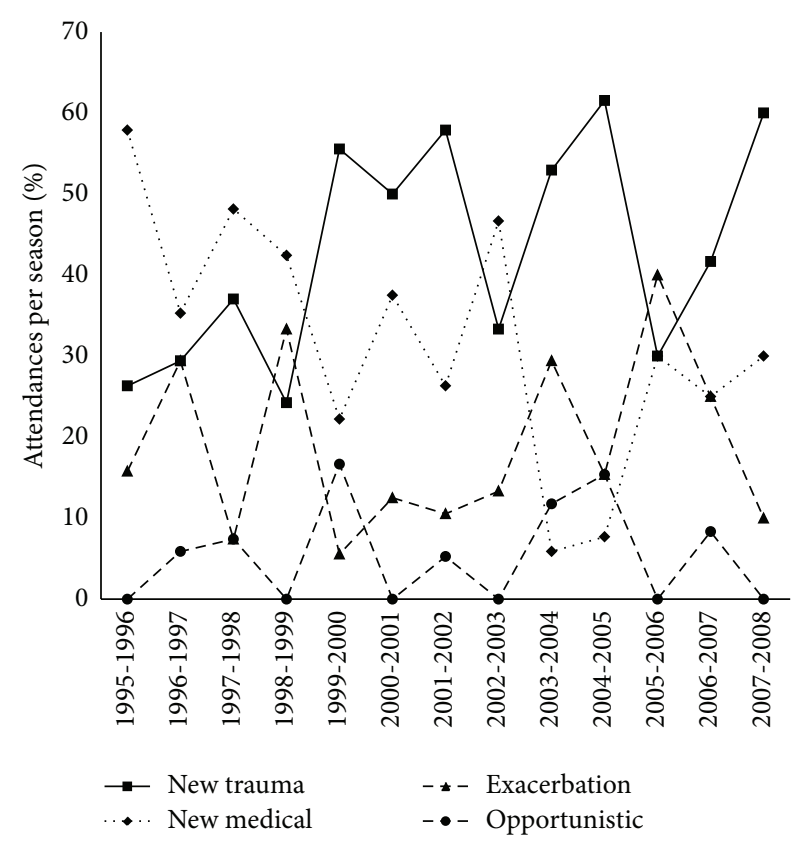

FIGURE 5: Trends in the reason for presentation to the crowd doctor over the study period for NTFC.

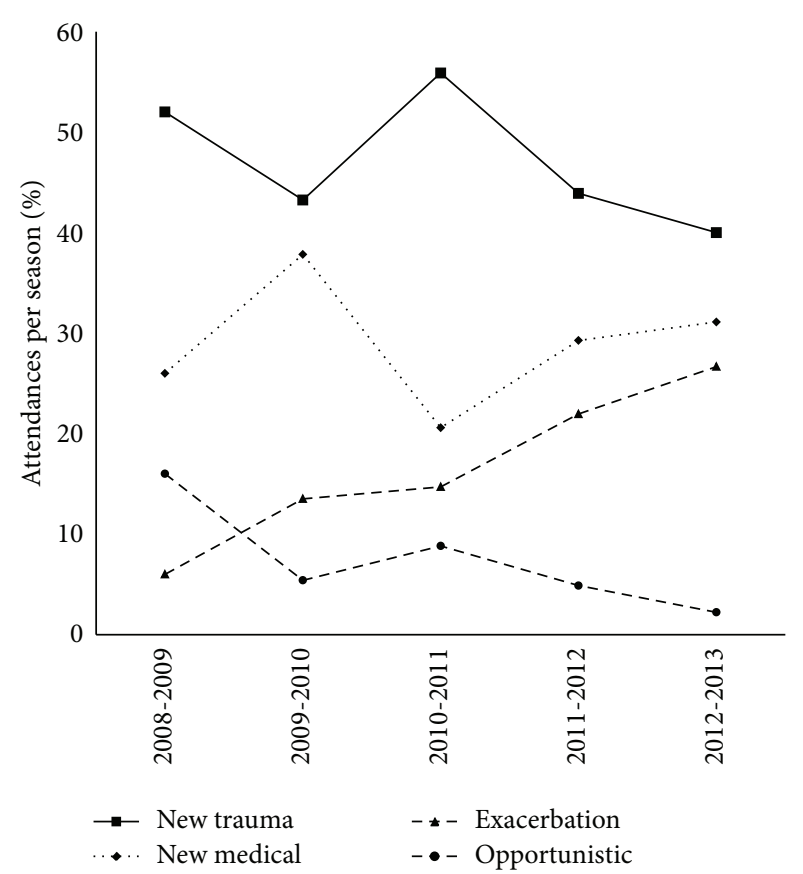

FIGURE 6: Trends in the reason for presentation to the crowd doctor over the study period for LCFC.

availability of the crowd doctors to seek medical advice for conditions that they should see their general practitioner or emergency department about. The vast majority of patients require only minor treatment and can safely return to the stadium. Presentation with serious, life-threatening conditions is thankfully rare, but on occasion the crowd doctor will be required to undertake a major intervention such as securing 
TABLE 2: Grading of severity of presentation and intervention of attendances to the crowd doctor. All values are percentages.

\begin{tabular}{lcccccc}
\hline & Minor & $\begin{array}{c}\text { Presentation } \\
\text { Intermediate }\end{array}$ & Major & Minor & \multicolumn{2}{c}{$\begin{array}{c}\text { Intervention } \\
\text { Intermediate }\end{array}$} \\
\hline Northampton & 79 & 19 & 3 & 88 & 11 & 1 \\
Leicester & 73 & 25 & 2 & 80 & 84 & 19 \\
\hline Overall & 76 & 21 & 2 & 84 & 15 \\
\hline
\end{tabular}

TABLE 3: Discharge destination of patients reviewed by crowd doctor.

\begin{tabular}{lcc}
\hline Discharge destination & $\begin{array}{c}\text { Number (percentage) } \\
\text { at NTFC }\end{array}$ & $\begin{array}{c}\text { Number (percentage) } \\
\text { at LCFC }\end{array}$ \\
\hline Returned to stadium & $130(58)$ & $114(56)$ \\
Returned to own home & $27(12)$ & $25(12)$ \\
Referred to hospital (immediate) & $38(17)$ & $28(14)$ \\
Referred to hospital (delayed) & $12(5)$ & $10(5)$ \\
Advised to see own GP & $19(8)$ & $19(9)$ \\
Mortuary & $0(0)$ & $1(0.5)$ \\
Declined hospital referral & $0(0)$ & $6(3)$ \\
\hline
\end{tabular}

the airway or CPR or treating myocardial ischaemia, and it is thus essential that crowd doctors are suitably trained and experienced in dealing with these conditions.

The rates of presentations seen at NTFC $(0.68-1.46$ presentations per 10,000 spectators) and LCFC (0.59-0.98 presentations per 10,000 spectators) are greater than those reported by the SGSA (approximately 0.4 presentations per 10,000) [4] but are broadly similar to the rates reported by Bhangu et al. and Crawford et al. in their analyses of single seasons at Aston Villa FC [6] and Glasgow Celtic FC [7] (both approximately 1.0 per 10,000 spectators). The rates are much lower than those reported by Leary et al. (3.1 per 10,000 spectators) at Millwall FC [5]. There is agreement between all papers that the majority of patients present with minor traumatic injuries requiring only minor assessment and treatment. In common with other studies, this paper reports a significant rate of opportunistic presentations. At Celtic, $21 \%$ of presentations were considered to be opportunistic, at Millwall the figure was $18 \%$, and at Aston Villa it was $10 \%$. This current study found that $5 \%$ of patients at NTFC and $8 \%$ of patients at LCFC presented with opportunistic presentations of conditions unrelated to attendance at the football match, whilst $19 \%$ of presentations at NTFC and $15 \%$ of presentations at LCFC were related to acute exacerbations of chronic diseases.

In this analysis, $21 \%$ of patients required transfer to hospital, either immediately or delayed by a few hours. This was a slightly higher rate than reported in other studies where rates of $9-15 \%$ were reported [5-7] and significantly higher than the $6 \%$ rate for all clubs reported by the SGSA [4].

The rate of presentations in which alcohol was considered a factor has shown wide variations between different clubs. It was deemed to be a contributing factor in $4 \%$ of cases at NTFC and $9 \%$ of cases at LCFC, broadly similar rates to those reported at Aston Villa (3\%) but significantly lower than the rate at Celtic (21\%).

The main strength of this study is the number of seasons analysed. Prior to this analysis, the most comprehensive paper was published by Leary et al. [5] who looked at six consecutive seasons at Millwall FC; this study looks at three times as many seasons and covers at least four million spectators (attendance data was unavailable for the first six seasons analysed). The data are likely to be reliable, as they were extracted from contemporaneous medical records, whose purpose was the documentation of history and examination findings and treatment administered in case of future legal action. The onus was thus on the doctor to ensure thorough documentation.

The limitations of this study are that the data were analysed retrospectively, and data were not recorded for the specific purpose of analysis at a later date. Consequently, the data did not take a standardised format and some interpretation was required when categorising the data. However, doublechecking of data classifications was performed independently by two authors to ensure consistency. Data were only available for the time periods when the authors worked as crowd doctors for the respective clubs. Consequently, no data are available for both clubs at the same time point and this has precluded direct comparisons between clubs. It is also possible that data were recorded inaccurately or incompletely, as evidenced by, for example, the large number of patients for whom no age or sex was recorded. Only presentations to the crowd doctor were analysed; casualties may have been seen at the stadium solely by other healthcare providers, for example, the St. John Ambulance or paramedics. However, personal experience suggests that the majority of patients presenting to these organisations are subsequently assessed by the crowd doctor. 
This analysis is important as it provides a long-term picture of crowd medical services at two football league clubs in England. It highlights that crowd doctors can expect to see patients in all age groups, including young children and the very elderly, presenting in a variety of ways, with traumatic injuries and acute medical and surgical problems, as well as with complications arising from their chronic disease states. Although the majority of patients seen will have only minor symptoms, requiring little more than basic analgesia or reassurance, a minority of patients will present with life-threatening problems. Consequently it is vital that the crowd doctor is comfortable assessing and treating all of these potential patient groups and presenting pathologies and this should be taken into account when considering the training and competence of doctors undertaking crowd medical coverage.

\section{Abbreviations}

CPR: Cardiopulmonary resuscitation

EMAS: East Midlands Ambulance Service

LCFC: Leicester City Football Club

NTFC: Northampton Town Football Club

SGSA: Sports Ground Safety Authority

UK: United Kingdom.

\section{Conflict of Interests}

The authors declare that there is no conflict of interests regarding the publication of this paper.

\section{Acknowledgments}

The authors would like to express their gratitude to all the individuals who have helped in the provision of medical coverage during their time as crowd doctors, in particular Dr. Maeve Kirwan (Crowd Doctor); Sue Robinson and Pauline Scoley (St. John's Ambulance); and Kevin Barclay (Stadium Safety Officer).

\section{References}

[1] R. Gibson, Report of the Medical Working Party, HMSO, London, UK, 1990.

[2] G. W. Kerr, S. C. Wilkie, and C. A. McGuffie, "Medical cover at Scottish football matches: have the recommendations of the Gibson Report been met?" British Journal of Sports Medicine, vol. 33, no. 4, pp. 274-275, 1999.

[3] Department of Culture Media and Sport, Guide to Safety at Sports Grounds, 2008, http://www.safetyatsportsgrounds.org .uk/sites/default/files/publications/green-guide.pdf.

[4] Injury Statistics, "Sports Grounds Safety Authority," http://www .safetyatsportsgrounds.org.uk/safety/injury-statistics.

[5] A. Leary, P. Greenwood, B. Hedley, J. Agnew, D. Thompson, and G. Punshon, "An analysis of use of crowd medical services at an English football league club," International Emergency Nursing, vol. 16, no. 3, pp. 193-199, 2008.

[6] A. Bhangu, C. Agar, L. Pickard, and A. Leary, "The Villa Park experience: crowd consultations at an English Premiership football stadium, season 2007-8," Emergency Medicine Journal, vol. 27, no. 6, pp. 424-429, 2010.

[7] M. Crawford, J. Donnelly, J. Gordon et al., "An analysis of consultations with the crowd doctors at glasgow celtic football club, season 1999-2000," British Journal of Sports Medicine, vol. 35, no. 4, pp. 245-249, 2001.

[8] Health Research Authority, "Defining research," 2013, http:// www.hra.nhs.uk/documents/2013/09/defining-research.pdf.

[9] Northampton Town Football Club Web site, http://www.ntfc .co.uk/.

[10] Leicester Football Club Web site, http://www.lcfc.com/. 


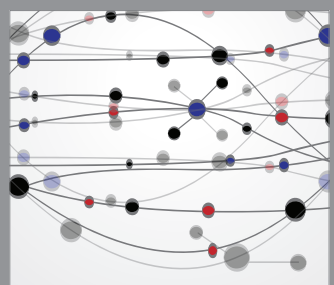

The Scientific World Journal
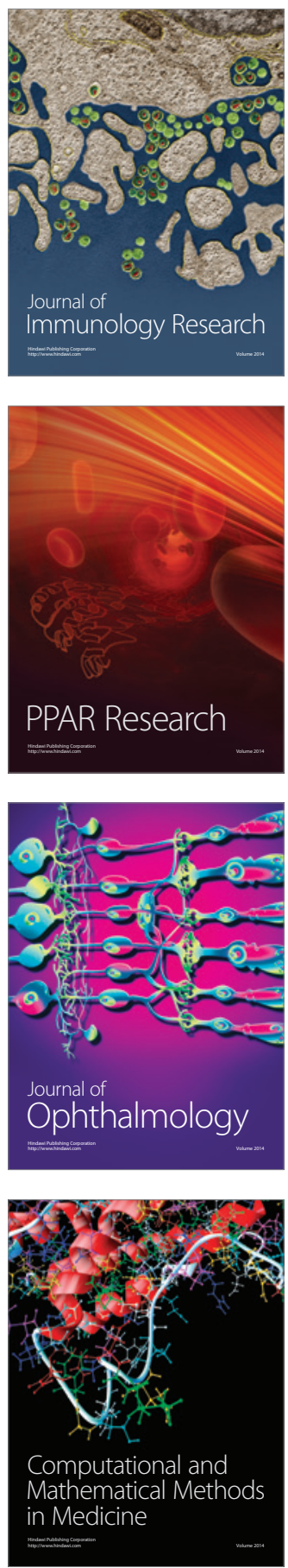

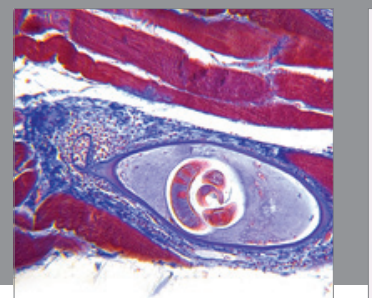

Gastroenterology

Research and Practice
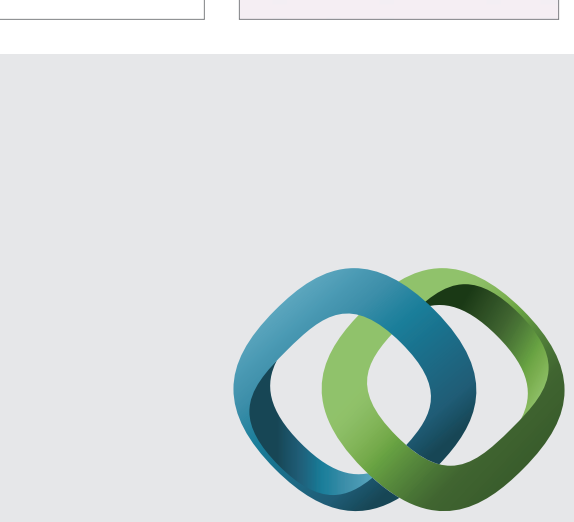

\section{Hindawi}

Submit your manuscripts at

http://www.hindawi.com
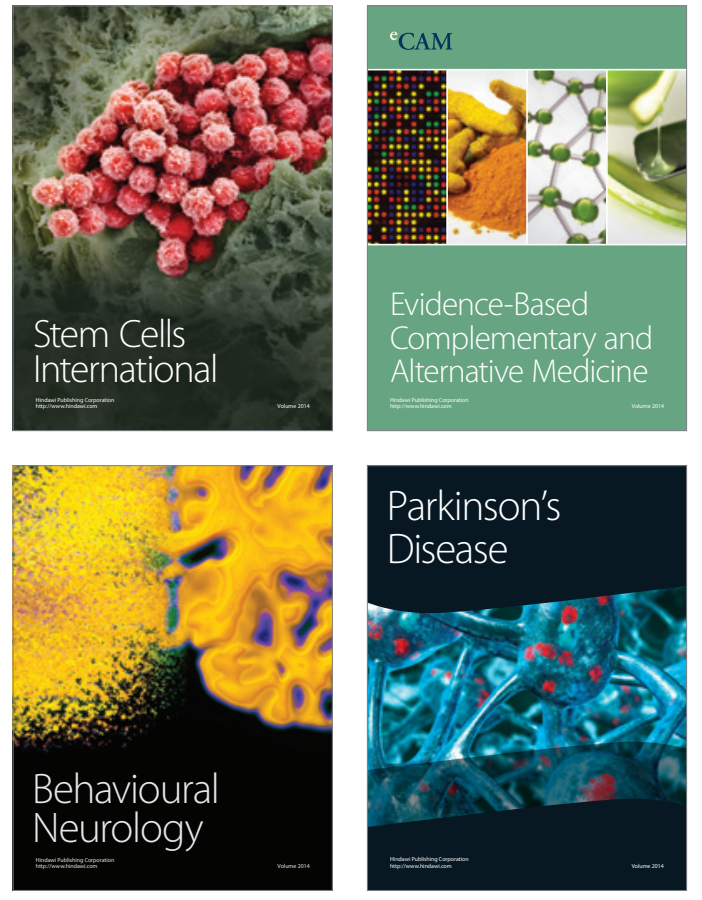
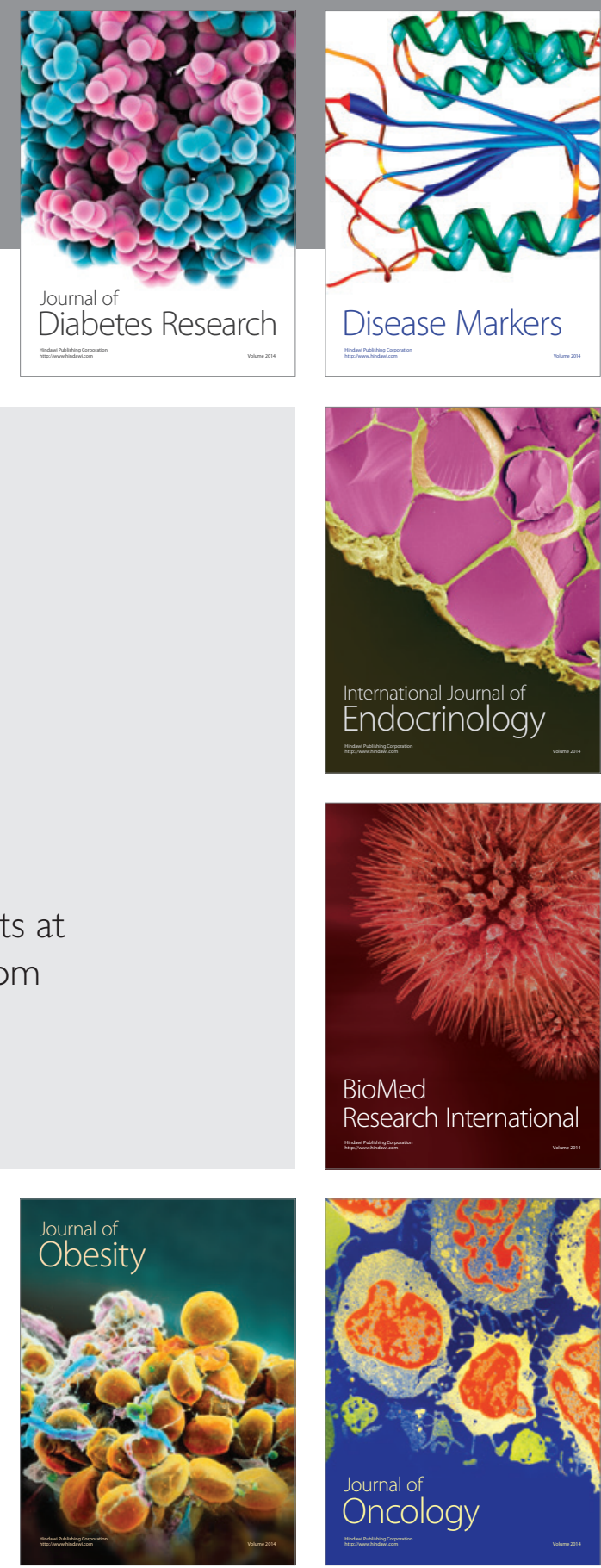

Disease Markers
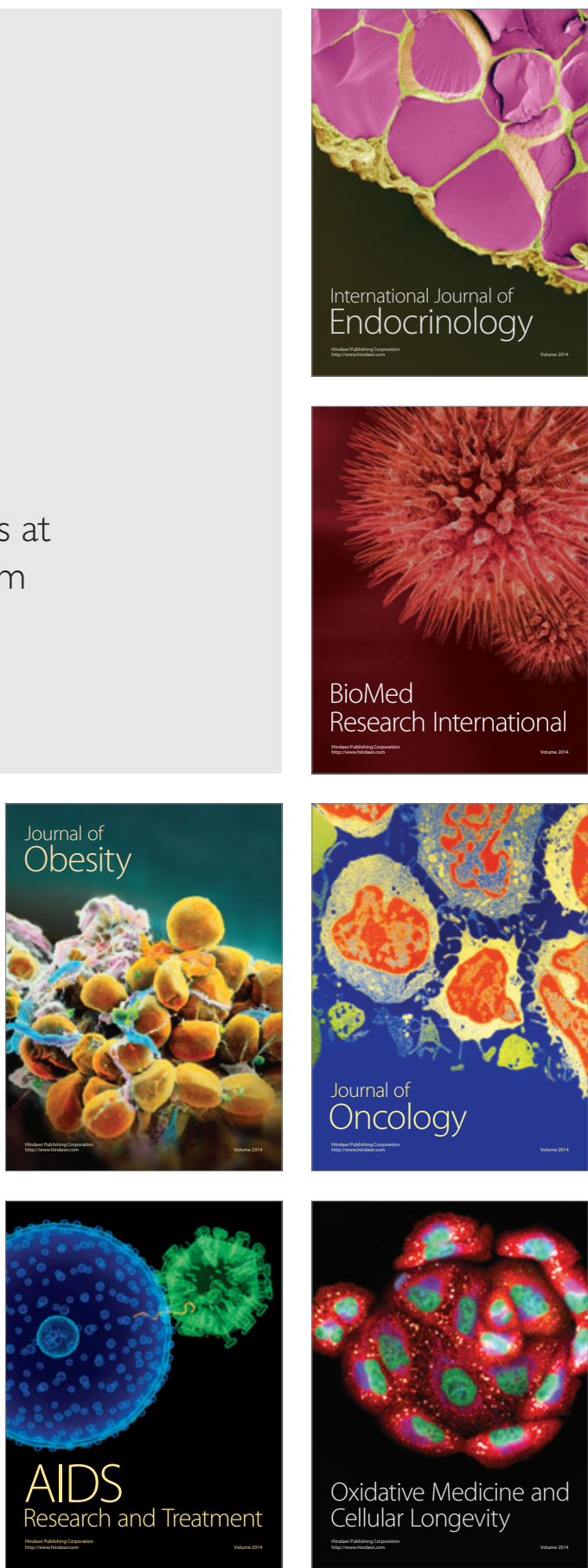